\title{
Second Generation of Moore-Read Quasiholes in a Composite Fermion Liquid
}

\author{
Arkadiusz Wójs ${ }^{1,2}$ Daniel Wodziński, ${ }^{1}$ and John J. Quinn ${ }^{2}$ \\ ${ }^{1}$ Institute of Physics, Wroclaw University of Technology, Wybrzeże Wyspiańskiego 27, 50-370 Wroclaw, Poland \\ ${ }^{2}$ Department of Physics, University of Tennessee, Knoxville, TN 37996, USA
}

\begin{abstract}
Two- and three-body correlations of incompressible quantum liquids are studied numerically. Pairing of composite fermions (CFs) in the 1/3-filled second CF Landau level is found at $\nu_{e}=4 / 11$. It is explained by reduced short-range repulsion due to ring-like single-particle charge distribution. Although Moore-Read state of CFs is unstable in the 1/2-filled second CF level, condensation of its quasiholes is a possible origin of incompressibility at $\nu_{e}=4 / 11$. Electron pairing occurs at $\nu_{e}=7 / 3$ and $13 / 3$, but with different pair-pair correlations. Signatures of triplets are found at higher fillings.
\end{abstract}

PACS numbers: 71.10.Pm, 73.43.-f

Strong magnetic field $B$ applied to a two-dimensional electron gas (2DEG) rearranges its single-particle density of states to a series of discrete Landau levels $\left(\mathrm{LL}_{n}\right)$. When the cyclotron gap $\hbar \omega_{c} \propto B$ exceeds Coulomb energy $e^{2} / \lambda \propto \sqrt{B}(\lambda=\sqrt{\hbar c / e B}$ being the magnetic length), the low-energy dynamics depends on interactions in one, partially filled LL. Despite reminiscence to atomic physics, macroscopic degeneracy and a distinct scattering matrix lead to very different, fascinating behavior [1].

Fractional quantum Hall effect 2] reveals plethora of highly correlated electron phases at various LL filling factors $\nu_{e}=2 \pi \varrho \lambda^{2}$ ( $\varrho$ being the $2 \mathrm{D}$ concentration). Among them are Laughlin [3] and Jain [4] incompressible liquids (IQLs) with fractionally charged quasiparticles (QPs) at $\nu_{e}=\frac{1}{3}$ or $\frac{2}{5}$, Wigner crystals [5] at $\nu_{e} \ll 1$, and stripes [6] in high LLs. Besides transport [2], they are probed by shot-noise (allowing detection of fractional charge of the QPs [] ) and optics (with discontinuities in photoluminescence energy related to the QP interactions [8]).

A key concept in understanding IQLs is Jain's composite fermion (CF) picture [4]. The CFs are fictitious particles, electrons that captured part of the external magnetic field $B$ in form of infinitesimal tubes carrying an even number $2 p$ of flux quanta $\phi_{0}=h c / e$. The most prominent IQLs at $\nu_{e}=n(2 p s \pm 1)^{-1}$ are represented by the completely filled $s$ lowest LLs of the CFs $\left(\mathrm{CF}-\mathrm{LL}_{n}\right.$ with $n<s)$ in a residual magnetic field $B^{*}=B-2 p \phi_{0} \varrho$.

Not all IQLs are so easily explained by the CF model, e.g., Haldane-Rezayi [9] and Moore-Read [10] paired liquids proposed for $\nu_{e}=\frac{5}{2}$. Because of nonabelian statistics of its quasiholes (QHs), especially the latter state has recently stirred renewed interest as a candidate for quantum computation in a solid-state environment 11].

Another family of IQLs discovered by Pan et al. 12] at $\nu_{e}=\frac{4}{11}, \frac{3}{8}$, and $\frac{5}{13}$ corresponds to fractional $\mathrm{CF}$ fillings $\nu_{\mathrm{CF}}=\nu_{e}\left(1-2 p \nu_{e}\right)^{-1}=\frac{4}{3}, \frac{3}{2}$, and $\frac{5}{3}$ (with $\left.p=1\right)$. Assuming spin polarization, all these states have a partially filled CF-LL $L_{1}$. Their incompressibility results from residual $\mathrm{CF}-\mathrm{CF}$ interactions. Familiar values of $\nu_{\mathrm{CF}}$ suggested similarity between partially filled electron and CF LLs 13. For $\nu_{e}=\frac{4}{11}$ and $\frac{5}{13}$, it revived the "QP hierarchy" 14], whose CF formulation consists of the $\mathrm{CF} \rightarrow$ electron

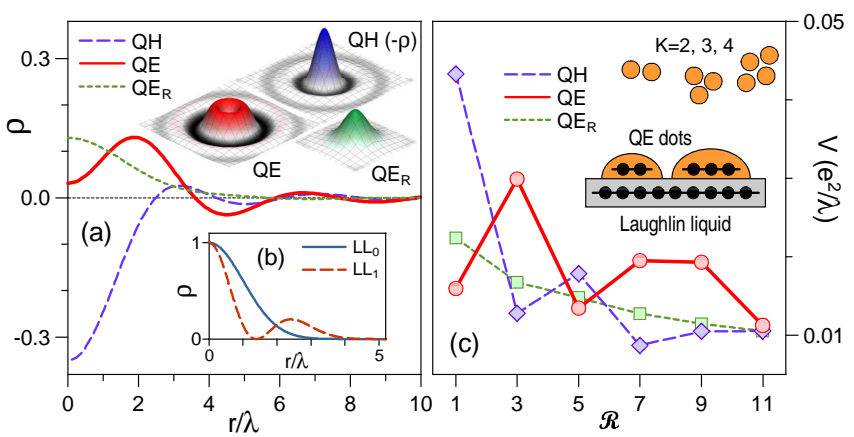

FIG. 1: (color online). (a) Radial charge distribution profiles of different composite fermions: Laughlin quasielectron (QE), quasihole $(\mathrm{QH})$, and reversed-spin quasielectron $\left(\mathrm{QE}_{\mathrm{R}}\right)$; results obtained from exact 10-electron diagonalization; $\lambda$ is the magnetic length. (b) Same for electrons in two lowest Landau levels. (c) Haldane pseudopotentials (interaction energy $V$ vs. relative pair angular momentum $\mathcal{R}$ ) for composite fermions; inset: schematic of "artificial composite fermion atoms."

mapping followed by reapplication of the $\mathrm{CF}$ picture in CF-LL $L_{1}$, leading to a "second generation" of CFs [15]. However, this idea ignored the requirement of a strong short-range repulsion [16, 17]. Indeed, it was later excluded in exact diagonalization studies [18], in which a different series of finite-size $\nu_{e}=\frac{4}{11}$ liquids with larger gaps was identified. On the other hand, Moore-Read liquid of paired CFs was tested [19] for $\nu_{e}=\frac{3}{8}$, but it was eventually ruled out in favor of the stripe order [20, 21].

In this Letter, we study two- and three-body correlations in several IQLs whose origin of incompressibility remains controversial. We find evidence for $\mathrm{CF}$ pairing in the $\nu_{e}=\frac{4}{11}$ liquid, hence interpreted as a condensate of (nonabelian) QHs of the "second generation" MooreRead state of the CFs. The pair-pair or $\mathrm{QH}-\mathrm{QH}$ correlations are not defined, but a Laughlin form is excluded.

In Fig. प $(\mathrm{a}, \mathrm{b})$ charge-density distributions of electrons are compared with three different $\mathrm{CF}$ quasiparticles at $\nu_{e}=\frac{1}{3}$. Laughlin liquid is a filled spin-polarized CF-LL $\mathrm{L}_{0}$, and its quasielectron $(\mathrm{QE})$, quasihole $(\mathrm{QH})$, and reversedspin quasielectron $\left(\mathrm{QE}_{\mathrm{R}}\right)$ correspond to a particle in $\mathrm{CF}$ $\mathrm{LL}_{1}$, a vacancy in CF-LL $\mathrm{L}_{0}$, and a spin-flip particle in CF- 
$\mathrm{LL}_{0}$, respectively. Particles/holes in CF-LL $\mathrm{L}_{0}$ resemble those in $\mathrm{LL}_{0}$. However, the ring structure in $\mathrm{CF}-\mathrm{LL}_{1}$ makes the QEs different from the electrons and causes strong reduction of the $\mathrm{QE}-\mathrm{QE}$ repulsion at short range [cf. Fig. 1(c)]. Such interaction cannot [16, 17] produce a Laughlin IQL of the QEs at the $\nu=\frac{1}{3}$ filling of CF-LL ${ }_{1}$. Instead, other $\mathrm{QE}-\mathrm{QE}$ correlations must be considered.

Spontaneous QE cluster formation would be somewhat analogous to the self-assembled growth of strained quantum dots 22. A full CF-LL $\mathrm{L}_{0}$ representing the uniformdensity Laughlin liquid plays the role of a "wetting layer." Over this background, in analogy to atoms grouping into dots to minimize the elastic energy, QEs moving within CF-LL $\mathrm{L}_{1}$ arrange themselves into pairs or larger QE clusters easily pinned down by disorder. While in electronic "artificial atoms" the self-organization of real atoms serves a purpose of external confinement for the electrons, in their CF analogs both these roles are played by the QEs. Another distinction is the fractional charge of bound QE carriers. A similar electron-atom analogy was earlier explored for condensation of cold atoms [23].

In numerics we considered $N \leq 12$ particles $(N=12$ being divisible by $K=2,3$, and 4 ) of charge $q$ ( $-e$ for electrons and $-\frac{1}{3} e$ for the $\mathrm{CFs}$ ) confined to a Haldane sphere [14] of radius $R$. For its high symmetry, this geometry is especially useful in studying quantum liquids. The radial magnetic field $B$ is created by a Dirac monopole of strength $2 Q=4 \pi R^{2} B \phi_{0}^{-1}$. The single-particle LLs are distinguished by shell angular momentum $l \geq Q$.

As for a partially filled atomic shell, the many-body hamiltonian on a sphere is determined by particle number $N$, shell degeneracy $g=2 l+1$, and interaction matrix elements. Using Clebsch-Gordan coefficients, the latter are related to Haldane 24] pseudopotentials $V_{L}$ (energies of pairs with angular momentum $L$ ). The pseudopotential combines information about the potential $v(r)$ and shell wavefunctions, so it may not be similar in different systems with the same (Coulomb) forces. In macroscopic quantum Hall systems, only the ratio $\nu=N / g$ (filling factor) is important, and $V$ is a function of relative pair angular momentum $\mathcal{R}=2 l-L$ (for fermions, an odd integer). The strategy in exact diagonalization is therefore to study different finite systems $(N, 2 l)$ with a realistic interaction $V(\mathcal{R})$, in search of those properties which scale properly with size and persist in the macroscopic limit.

In the following we will distinguish $\nu_{e}=2 \pi \varrho \lambda^{2}$ from the effective filling factor $\nu=N / g<1$ of only those electrons or CFs in their highest, partially filled shell.

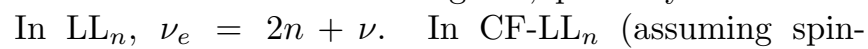
polarization) $\nu_{\mathrm{CF}}=n+\nu$ and $\nu_{e}=\nu_{\mathrm{CF}}\left(2 p \nu_{\mathrm{CF}}+1\right)^{-1}$.

The CF pseudopotentials shown in Fig. 1(c) were obtained using a similar method to Ref. 20], by combining short-range data from exact diagonalization [17] with long-range behavior of point charges $\pm \frac{1}{3} e$. Weak $\mathrm{QE}-$ $\mathrm{QE}$ repulsion at $\mathcal{R}=1$ is the reason why the $\nu=\frac{1}{3}$, $\frac{2}{3}$, and $\frac{1}{2}$ states of QEs are not the "second generation"

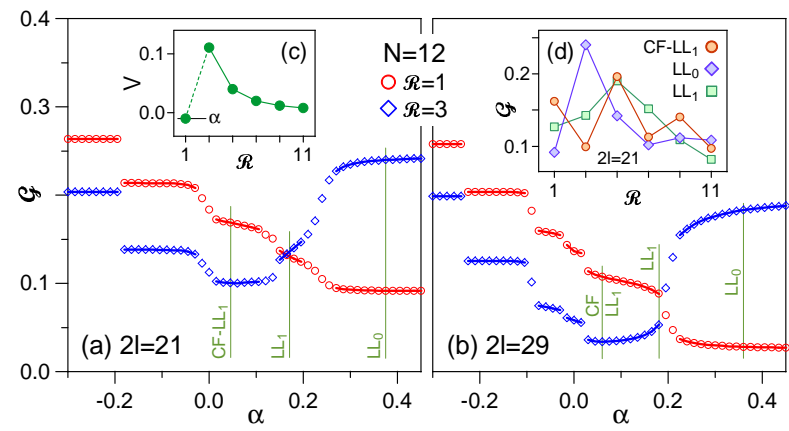

FIG. 2: (color online). Haldane pair amplitudes $\mathcal{G}(\sim$ number of pairs) at relative pair angular momenta $\mathcal{R}=1$ and 3 , of $N=12$ fermions in angular momentum shells with $2 l=21$ (a) and $2 l=29(\mathrm{~b})$, as a function of parameter $\alpha$ of the interaction pseudopotential shown in (c). (d) Amplitudes $\mathcal{G}(\mathcal{R})$ of electrons and composite fermions in different Landau levels.

Laughlin, Jain, or Moore-Read states (of QEs). The average $\mathrm{QE}-\mathrm{QE}$ interaction energies (per particle) in these states overestimates the actual QE eigenenergies by at least $0.003 e^{2} / \lambda(6-7 \%)$. Clearly, the microscopic origin of the observed QE incompressibility must be different.

What are these known correlations, excluded for QEs? Laughlin correlations result from strong short-range repulsion (such as between electrons in $\mathrm{LL}_{0}$ ). They consist of the maximum avoidance of pair states with the smallest $\mathcal{R}$. E.g., Laughlin $\nu=\frac{1}{3}$ state is the zero-energy ground state of a model pseudopotential $V=\delta_{\mathcal{R}, 1}$ [14]. For more realistic interactions, the exact criterion is that $V$ must rise faster than linearly when $\mathcal{R}$ decreases 17 . A linear decrease of $V$ between $\mathcal{R}=1$ and 5 (such as in $\mathrm{LL}_{1}$ ) leads to different correlations. E.g., Moore-Read $\nu=\frac{1}{2}$ liquid involves pairing and Laughlin correlations among pairs. It is the zero-energy ground state of a model threebody pseudopotential $V=\delta_{\mathcal{T}, 3}(\mathcal{T}=3 l-L \geq 3$ is the relative triplet angular momentum, proportional to the area spanned by three particles) [10].

Weak QE-QE repulsion at $\mathcal{R}=1$ compared to $\mathcal{R}=3$ could force QEs into even larger clusters. As a simple classical analogy, consider a string of point particles, one per unit length, with a repulsive potential $v_{a}(r)=a+(1-a) r$ for $r<1$ and $1 / r^{2}$ otherwise. Equal spacing is favored for $a>1.64$, and transitions to pairs, triplets, and larger clusters occur for decreasing $a$. A similar rearrangement might occur when going from $\mathrm{LL}_{0}$ to $\mathrm{LL}_{1}$ and CF-LL ${ }_{1}$, with $V(1)$ playing the role of $v_{a}(0) \equiv a$.

In Fig. 2 we plot two leading "Haldane amplitudes" 24], $\mathcal{G}(1)$ and $\mathcal{G}(3)$. The discrete pair-correlation function $\mathcal{G}(\mathcal{R})$ is proportional to the number of pairs with a given $\mathcal{R}$ and normalized to $\sum_{\mathcal{R}} \mathcal{G}(\mathcal{R})=1$. It connects many-body interaction energy with a pseudopotential, $E=\left(\begin{array}{c}N \\ 2\end{array}\right) \sum_{\mathcal{R}} \mathcal{G}(\mathcal{R}) V(\mathcal{R})$. Here, $\mathcal{G}$ is calculated in the ground states of $N=12$ particles at $2 l=21$ and 29 (corresponding to $\nu=\frac{1}{2}$ and $\frac{1}{3}$ for the QEs [18]) with 


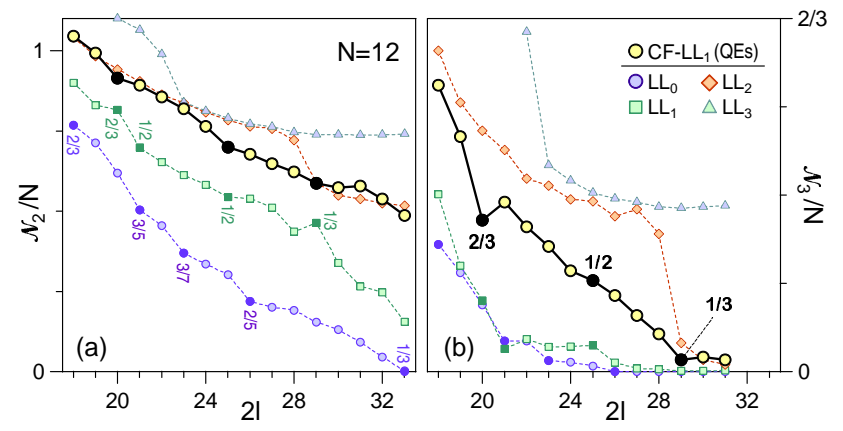

FIG. 3: (color online). Number of pairs $\mathcal{N}_{2}$ (a) and triplets $\mathcal{N}_{3}$ (b) with the minimum relative angular momentum $(\mathcal{R}=1$ or $\mathcal{T}=3$ ) for $N=12$ electrons or composite fermions in angular momentum shells with $2 l=18$ to 33, corresponding to fractional Landau level fillings $\frac{1}{3} \leq \nu \sim N /(2 l+1) \leq \frac{2}{3}$. Finite-size incompressible states are labeled by $\nu$.

model interaction shown in the inset: $V_{\alpha}(1)=\alpha$ and $V_{\alpha}(\mathcal{R}>1)=1 / \mathcal{R}^{2}$. At $\alpha>0.3, \mathcal{G}(1)$ takes on the minimum possible value, which means Laughlin correlations (no clusters). At $\alpha<-0.25, \mathcal{G}(1)$ reaches maximum, and the particles form one big $\nu=1$ quantum Hall droplet (QHD). The transition between the two limits occurs quasi-discontinuously through a series of welldefined states seen as plateaus in $\mathcal{G}(\alpha)$.

The cluster size $K$ cannot be assigned to each state because the number of plateaus depends on the choice of $V_{\alpha}$. The comparison of $\mathcal{G}(1)$ with the values predicted for $N / K$ independent QHDs of size $K=2,3$, and 4 is not convincing because in a few-cluster system each QHD is relaxed by the cluster-cluster interaction, lowering $\mathcal{G}(1)$. Another problem is the contribution to $\mathcal{G}(1)$ from pairs of particles belonging to different clusters. Nevertheless, it is clear that the "degree of clustering" changes as a function of $\alpha$ in a quantized fashion, supporting the picture of $N$ particles grouping into various clustered configurations. Furthermore, the values of $\alpha$ for which $V_{\alpha}$ reproduces the exact ground states of QEs or electrons belong to different continuity regions, confirming different correlations in $\mathrm{LL}_{0}, \mathrm{LL}_{1}$, and CF-LL $\mathrm{LL}_{1}$ (except for a possible similarity of the $\nu=\frac{1}{3}$ states in $\mathrm{LL}_{1}$ and CF-LL $\mathrm{LL}_{1}$ ).

In Fig. 3(a) we compare $\mathcal{N}_{2}=\left(\begin{array}{c}N \\ 2\end{array}\right) \mathcal{G}(1)$, the number of pairs with $\mathcal{R}=1$, calculated in the ground states of $N=$ $12 \mathrm{CFs}$ and electrons as a function $2 l$. The downward cusps in $\mathcal{N}_{2}(2 l)$ at a series of Laughlin/Jain states in $\mathrm{LL}_{0}$ are well understood. We also marked $2 l=2 N-3$ and $3 N-7$ corresponding to incompressible $\nu=\frac{1}{2}$ and $\frac{1}{3}$ ground states in $\mathrm{LL}_{1}$ and CF-LL 18 , and their particlehole conjugates $(N \rightarrow g-N)$ at $2 l=2 N+1$ and $\frac{3}{2} N+2$.

The comparison of $\mathcal{N}_{2}$ tells about short-range pair correlations in different LLs. There are significantly more pairs in CF-LL $L_{1}$ and in excited electron LLs than in $\mathrm{LL}_{0}$. In $\mathrm{LL}_{1}$, the Moore-Read state is known to be paired, and indeed $\mathcal{N}_{2} \approx \frac{1}{2} N$ at $\nu=\frac{1}{2}$. A similar value is obtained for the (not well understood) $\nu=\frac{1}{3}$ state at $2 l=29$. The CF-LL $L_{1}$ is different (in terms of $\mathcal{N}_{2}$ ) from $L_{0}$ or $L_{1}$ in the whole range of $18 \leq 2 l \leq 33$. However, it appears similar to $\mathrm{LL}_{2}$ at both $2 l \leq 23$ and $2 l \geq 29$. Also, $\mathrm{LL}_{2}$ and $\mathrm{LL}_{3}$ look alike for $23 \leq 2 l<29$. While convincing assignment of $\nu$ to a finite state $(N, 2 l)$ requires studying size dependence (we looked at different $N \leq 12$ ), notice that $N / g=\frac{1}{2}$ at $2 l=23$, and $2 l=29$ is the $\nu=\frac{1}{3}$ state in $\mathrm{LL}_{1}$ and CF-LL ${ }_{1}$. Note also that similar shortrange correlations do not guarantee high wavefunction overlaps. Here, only $\left\langle\mathrm{LL}_{2} \mid \mathrm{LL}_{3}\right\rangle^{2}$ reaches 0.67 while all other overlaps, including $\left\langle\mathrm{QE} \mid \mathrm{LL}_{n}\right\rangle^{2}$, essentially vanish.

In Fig. 31(b) we plot $\mathcal{N}_{3}$, the number of "compact" triplets with $\mathcal{T}=3$. It is proportional to the first triplet Haldane amplitude and tells about short-range three-body correlations. In both $\mathrm{LL}_{0}$ and $\mathrm{LL}_{1}, \mathcal{N}_{3}$ decreases roughly linearly as a function of $2 l$ and drops to essentially zero at $2 l=21$, the smallest value at which the $\mathcal{T}=3$ triplets can be completely avoided. Exactly $\mathcal{N}_{3}=0$ would indicate the Moore-Read state, but its accuracy for the actual $\nu=\frac{1}{2}$ ground state in $\mathrm{LL}_{1}$ depends sensitively on the quasi-2D layer width and on the surface curvature. Nevertheless, clusters larger than pairs clearly do not form in neither $\mathrm{LL}_{0}$ nor $\mathrm{LL}_{1}$ at $\nu \leq \frac{1}{2}$.

The number of QE triplets in CF-LL $L_{1}$ is also a nearly linear function of $2 l$, but it drops to zero at $2 l=3 N-7=$ 29, earlier identified with $\nu=\frac{1}{3}$ in this shell (i.e. with $\left.\nu_{e}=\frac{4}{11}\right)\left[18\right.$. In connection with having $\mathcal{N}_{2} \approx \frac{1}{2} N$ pairs, vanishing of $\mathcal{N}_{3}$ is the evidence for $Q E$ pairing at $\nu_{e}=\frac{4}{11}$.

The elementary excitations that appear in the paired $\nu=\frac{1}{2}$ Moore-Read state when $2 l>2 N-3$ are the $\frac{1}{4} q$ charged QHs (of the Laughlin liquid of pairs) and pairbreaking neutral-fermion excitations [10]. Being paired, the QE state at $2 l=3 N-7$ can only contain the QHs but no pair-breakers. The interaction of Moore-Read QHs in $\mathrm{CF}-\mathrm{LL}_{1}$ is not known, but evidently it causes their condensation into an incompressible liquid at $\nu=\frac{1}{3}$.

The "second generation" (to distinguish from $\nu_{e}=\frac{5}{2}$ ) Moore-Read state of QEs would occur at $\nu=\frac{1}{2}$ in CF$\mathrm{LL}_{1}$ (i.e., at $\nu_{\mathrm{CF}}=\frac{3}{2}$ or $\nu_{e}=\frac{3}{8}$ ). Its instability [20, 21] does not preclude reentrance with additional QHs at a lower $\nu$ and, in particular, their condensation at $\nu=\frac{1}{3}$ (i.e., at $\nu_{\mathrm{CF}}=\frac{4}{3}$ or $\nu_{e}=\frac{4}{11}$ ). A similar situation occurs with Jain $\nu=\frac{2}{7}$ state, obtained (in Haldane hierarchy) from Laughlin $\nu=\frac{1}{3}$ state by addition of "second generation" Laughlin QHs. There, stability of the $\nu=\frac{2}{7}$ daughter does not require stability of the $\nu=\frac{1}{3}$ parent.

The value of $2 l=3 N-7$ precludes a Laughlin state of pairs (or, equivalently, of the QHs). To show it, let us use the following pictorial argument, equivalent to a more rigorous derivation. Laughlin $\nu=\frac{1}{3}$ state (of individual particles) can be viewed as $\bullet \circ \bullet \bullet . . \bullet \circ \bullet \equiv(\bullet \circ) \bullet$, with "•" and "o" denoting particles and vacancies. Counting the total LL degeneracy $g$ leads to the correct value of $2 l=3 N-3$. The Moore-Read state, i.e., the Laughlin state of pairs at $\nu=\frac{1}{2}$, is represented by 


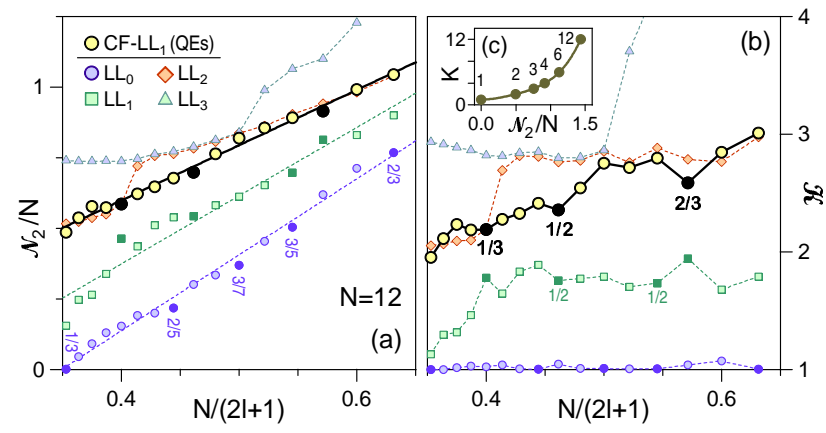

FIG. 4: (color online). Number of pairs $\mathcal{N}_{2}$ with the minimum relative angular momentum $\mathcal{R}=1$ (a) and estimated average cluster size $\mathcal{K}$ (b) for $N=12$ electrons or composite fermions in Landau levels angular momentum shells with $2 l=18$ to 33, plotted as a function of the filling factor $\nu \sim N /(2 l+1)$.

$(\bullet \bullet \circ) \bullet \bullet$, yielding $2 l=2 N-3$. A Laughlin state of pairs at $\nu=\frac{1}{3}$ would correspond to $(\bullet \bullet \circ \circ \circ) \bullet \bullet$, predicting (incorrectly) $2 l=3 N-5$. Assumming pairing, $2 l=3 N-7$ can only be obtained using a two-pair unit cell, $(\bullet \bullet \circ 0 \bullet \bullet \circ 00000) \bullet \bullet \circ \bullet \bullet \bullet$, corresponding to more complicated pair-pair correlations.

At higher fillings of CF-LL $1, \mathcal{N}_{3} \approx \frac{1}{3} N$ at $2 l=20$ suggests division of $N$ QEs into $\frac{1}{3} N$ triplets at $\nu=\frac{2}{3}$, and $\mathcal{N}_{3} \approx \frac{1}{6} N$ at $2 l=25$ implies a more complicated cluster configuration (with mixed sizes) at $\nu=\frac{1}{2}$. $\mathrm{LL}_{2}$ and $\mathrm{LL}_{3}$ look alike (and different from $\mathrm{LL}_{0}$ or CF-LL $\mathrm{L}_{1}$ ) at $23 \leq 2 l<29$, both having $\mathcal{N}_{3} \approx \frac{1}{3} N$. At $2 l=29, \mathcal{N}_{3}$ for $\mathrm{LL}_{2}$ drops rapidly to almost zero. This further supports similarity of the $\nu=\frac{1}{3}$ states in $\mathrm{LL}_{2}$ and CF-LL $\mathrm{LL}_{1}$

In Fig. 廿(a) we replot $\mathcal{N}_{2}$ as a function of $N / g \sim \nu$. The quasi-linear dependences for $\mathrm{LL}_{0}, \mathrm{LL}_{1}$, and CF-LL all aim correctly at $\mathcal{N}_{2}=2 N-3$ for $\nu=1$, but start from different values, $\mathcal{N}_{2} \approx 0, \frac{1}{4} N$, and $\frac{1}{2} N$, at $\nu=\frac{1}{3}$. Regular dependence allows subtraction from $\mathcal{N}_{2}$ the contribution from pairs belonging to different clusters. As reference we used ground states of $V=\delta_{\mathcal{R}, 1}$. This short-range repulsion guarantees maximum avoidance of $\mathcal{R}=1$; its $\mathcal{N}_{2}^{*}$ contains only the inter-cluster contribution. To compare $\mathcal{N}_{2}$ of QEs or electrons with $\mathcal{N}_{2}^{*}$, we: (i) calculated $\mathcal{N}_{2}$ for a single $K$-size cluster, and multiplied it by $N / K$ to obtain relation between $\mathcal{N}_{2}$ and $K$ in an idealized clustered state of $N$ particles, (ii) using this relation [cf. Fig. [4(c)], converted $\mathcal{N}_{2}$ and $\mathcal{N}_{2}^{*}$ into the (average) cluster sizes $K$ and $K^{*}$; (iii) defined $\mathcal{K}=K-\left(K^{*}-1\right)$ as the cluster size estimate free of the inter-cluster contribution.

The result in Fig. 目(b) indicates pairing in $\mathrm{LL}_{1}$ at $\frac{1}{3} \leq$ $\nu \leq \frac{2}{3}$, and in both CF-LL $L_{1}$ and $\mathrm{LL}_{2}$ at $\nu \leq \frac{1}{3}$. Triplets seem to form in CF-LL 1 at $\nu=\frac{2}{3}$, in $\mathrm{LL}_{2}$ at $\frac{1}{3} \leq \nu \leq \frac{2}{3}$, and in $\mathrm{LL}_{3}$ at $\nu \leq \frac{1}{2}$. The $\nu=\frac{1}{2}$ state of QEs falls between $\mathcal{K}=2$ and 3, suggesting mixed-size clusters.

In conclusion, we studied two- and three-body correlations of several quantum liquids. We found evidence for pairing of CFs at $\nu_{e}=\frac{4}{11}$ and interpret this state as a condensate of "second generation" Moore-Read QHs.
AW thanks Wei Pan and Jan Jaroszyński for helpful discussions. Work supported by DOE Basic Energy Science and Grant 2P03B02424 of the Polish MENiS.

[1] D. Yoshioka, The Quantum Hall Effect (Springer-Verlag, New York, 2002), and references therein.

[2] D. C. Tsui, H. L. Störmer, and A. C. Gossard, Phys. Rev. Lett. 48, 1559 (1982); R. Willett, J. P. Eisenstein, H. L. Störmer, D. C. Tsui, A. C. Gossard, and J. H. English, ibid. 59, 1776 (1987).

[3] R. B. Laughlin, Phys. Rev. Lett. 50, 1395 (1983).

[4] J. K. Jain, Phys. Rev. Lett. 63, 199 (1989); Science 266, 1199 (1994); Phys. Today 53, No. 4, 39 (2000).

[5] Y.E. Lozovik and V.I. Yudson, JETP Lett. 22, 11 (1975).

[6] A. A. Koulakov, M. M. Fogler, and B. I. Shklovskii, Phys. Rev. Lett. 76, 499 (1996); E. H. Rezayi, F. D. M. Haldane, and Kun Yang, ibid. 83, 1219 (1999).

[7] L. Saminadayar et al., Phys. Rev. Lett. 79, 2526 (1997); R. De-Picciotto et al., Nature (London) 389, 162 (1997).

[8] A. J. Turberfield et al., Phys. Rev. Lett. 65, 637 (1990); B. B. Goldberg et al., ibid. 65, 641 (1990); M. Byszewski et al., Nature Physics (London) 2, 239 (2006).

[9] F. D. M. Haldane and E. H. Rezayi, Phys. Rev. Lett. 60, 956 (1988).

[10] G. Moore and N. Read, Nucl. Phys. B 360, 362 (1991); M. Greiter, X.-G. Wen, and F. Wilczek, Phys. Rev. Lett. 66, 3205 (1991); V. W. Scarola, K. Park, and J. K. Jain, Nature (London) 406, 863 (2000).

[11] S. Das Sarma, M. Freedman, and C. Nayak, Phys. Rev. Lett. 94, 166802 (2005).

[12] W. Pan, H. L. Störmer, D. C. Tsui, L. N. Pfeiffer, K. W. Baldwin, and K. W. West, Phys. Rev. Lett. 90, 016801 (2003).

[13] S. S. Mandal and J. K. Jain, Phys. Rev. B 66, 155302 (2002).

[14] F. D. M. Haldane, Phys. Rev. Lett. 51, 605 (1983).

[15] J. H. Smet, Nature (London) 422, 391 (2003); C.-C Chang and J. K. Jain, Phys. Rev. Lett. 92, 196806 (2004); M. R. Peterson and J. K. Jain, ibid. 93, 046402 (2004); M. O. Goerbig, P. Lederer, and C. Morais Smith, ibid. 93, 216802 (2004).

[16] F. D. M. Haldane and E. H. Rezayi, Phys. Rev. Lett. 54, 237 (1985).

[17] A. Wójs and J. J. Quinn, Phys. Rev. B 61, 2846 (2000).

[18] A. Wójs, K.-S. Yi, and J. J. Quinn, Phys. Rev. B 69, 205322 (2004); A. Wójs, D. Wodziński, and J. J. Quinn, ibid. 71, 245331 (2005).

[19] K. Park, V. Melik-Alaverdian, N. E. Bonesteel, and J. K. Jain Phys. Rev. B 58, R10167 (1998).

[20] S.-Y. Lee, V. W. Scarola, and J. K. Jain, Phys. Rev. Lett. 87, 256803 (2001); Phys. Rev. B 66, 085336 (2002).

[21] N. Shibata and D. Yoshioka, J. Phys. Soc. Jpn. 73, 1 (2004); ibid. 73, 2169 (2004).

[22] L. Jacak, P. Hawrylak, and A. Wójs, Quantum Dots (Springer-Verlag, Berlin, 1998), and references therein.

[23] N. K. Wilkin and J. M. F. Gunn, Phys. Rev. Lett. 84, 6 (2000).

[24] F. D. M. Haldane, in: The Quantum Hall Effect, edited by R. E. Prange and S. M. Girvin, (Springer-Verlag, New York, 1987), chapter 8, pp. 303-352. 